DEGENERATE DYNAMICAL SYSTEMS AND THE DISAPPEARANCE OF (K.A.M.-TYPE) INTEGRALS OF MOTION

\title{
H. VARVOGLIS
}

ASTRONOMY DEPARTMENT, UNIVERSITY OF THESSALONIKI, GREECE.

\section{ABSTRACT}

The problem of constructing the stochasticity criterion of a degenerate near-integrable dynamical system is considered. We show that in certain cases the above criterion can be found as the limit of the corresponding stochasticity criteria of a family of non-degenerate systems, whose limit is the degenerate one.

\section{INTRODUCTION}

The knowledge of the stable or unstable nature of the trajectories in a certain region of a dynamical system's phase space is of paramount importance in many cases of interest in Celestial Mechanics. More specifically regions of stability are suitable for applications of the theory of the KAM-type integrals of motion (mainly for long time predictions for the evolution of the system) while regions of instability are suitable for a statistical approach (based on the assumed ergodicity of the corresponding trajectories). In the latter case the unstable nature of the trajectories has been used to explain various phenomena, as the Kirkwood gaps in the asteroid belt (e.g., see Berry 1978, Hadjidemetriou and Ichtiaroglou 1983), particle acceleration by waves (e.g. Fukuyama et al. 1977) etc.

It is by now generally accepted that not only dynamical systems differing solely in the numerical value of a parameter can show either stable or unstable behavior as described above, but that even the same system may show different stability characteristics in different regions of phase space. The existence of a criterion (usually called "stochasticity criterion") that can give the phase space regions of stability and instability (regions of order and chaos respectively) of a certain perturbed integrable dynamical system as a function of the strength of the perturbation is of obvious importance. It has been shown (Chirikov 1969, 1979) that in the case of a non-degenerate two dimensional Hamiltonian system of the form (Arnold 1978)

$$
\mathrm{H}=\mathrm{H}_{0}\left(I_{1}, I_{2}\right)+\varepsilon \mathrm{H}_{1}\left(I_{1}, I_{2}, \theta_{1} \theta_{2}\right)
$$

such a criterion can be constructed by a simple reasoning where by non411

V. V. Markellos and Y. Kozai (eds), Dynamical Trapping and Evolution in the Solar System, 411-41S.

() 1983 by D. Reidel Publishing Company. 
degenerate we mean that the frequencies of the "unperturbed" $\left(\mathrm{H}=\mathrm{H}_{0}\right)$ Hamiltonian function depend on the actions, that is

$$
\left|\frac{\partial^{2} H_{0}}{\partial I_{i} \partial I_{j}}\right| \neq 0 \quad \text { or } \quad\left|\begin{array}{cc}
\frac{\partial^{2} H_{0}}{\partial I_{i} I_{j}} & \frac{\partial H_{0}}{\partial I_{i}} \\
\frac{\partial H_{0}}{\partial I_{j}} & 0
\end{array}\right| \neq 0
$$

Many of the systems of interest, however, are degenerate, as it is for instance the case with the well known Henon and Heiles (1965) and Contopoulos-Barbanis (Barbanis 1966) potentials. To find a stochasticity criterion in the case of a degenerate system seems to be a more involved problem. It is the purpose of this work to show, using a specific example, that the correct stochasticity criterion of a degenerate dynamical system can be found in certain cases by considering the limit of the corresponding criteria of a family of non-degenerate systems.

III. THE EXAMPLE CASE

Consider the Hamiltonian system

$$
\mathrm{H}_{0}=\mathrm{H}_{0}+\varepsilon \mathrm{H}_{1}=\mathrm{I}_{1}+\nu \mathrm{I}_{2}+\frac{1}{2} \xi^{2} I_{2}{ }^{2}-\varepsilon \sum_{\ell=-\infty}^{\infty} J_{\ell}\left\{\left(2 I_{1}\right)^{1 / 2}\right\} \sin \left(\ell \theta_{1}-\theta_{2}\right)
$$

which has the following properties:

i) for $\varepsilon=0$ it is completely integrable, so that it does not show stochastic behavion at all.

ii) for $\varepsilon>0$ but $\varepsilon<\varepsilon_{C}\left(\nu^{*}, \xi\right)$ it behaves like an integrable one (the KAM-type integral of motion exists in most of phase space).

iii) for $\varepsilon>\varepsilon_{C}(\nu *, \xi)$ there are regions in phase space where the system shows large scale chaotic behaviour and the corresponding trajectories are intrinsically unstable. The value of $\varepsilon_{c}$ is, as noted above, a function of $\nu^{*}$ and $\xi$, where $\nu^{*}=\nu+\xi I_{2}$ is the inverse of the rotation number of $\mathrm{H}_{0}$ and the parameter $\xi$ gives the degree of non-degeneracy of the system, If $\xi=0$ we have

$\omega_{1}=\frac{\partial \mathrm{H}_{0}}{\partial \mathrm{I}_{1}}=1, \quad \omega_{2}=\frac{\partial \mathrm{H}_{0}}{\partial \mathrm{I}_{2}}=v$

and the dynamical system is degenerate; if $\xi>0$ the degeneracy is removed.

When $\xi \simeq 1$ the stochasticity criterion for equ. ( 3 ) has been found (Smith and Kaufman 1978) to be

$$
J_{n *}\left\{\left(2 I_{1}\right)^{1 / 2}\right\} \geqslant \frac{1}{16 \varepsilon \xi^{2}}
$$

where $n *$ is the integer nearest to $\nu^{*}$. When $\xi=0$ on the other hand the stochasticity criterion is (Fukuyama et al. 1977, Hsu 1982) 


$$
\frac{\left\{I_{n}\left(2 I_{1}\right)^{1 / 2}\right\}}{\left(2 I_{1}\right)^{1 / 2}} \geqslant \frac{1}{4 n \varepsilon}
$$

where $\mathrm{n}$ is the integer nearest to $\nu$ again.

Despite the fact that the chaos sets on in a different way when $\xi \simeq 1$ or $\xi=0$, the similarity in the form of equs. (4) and (5) leads one to investigate whether these equations could be obtained as special cases of a more general relation. To do so at first we apply the usual arguments (e.g., Greene 1980) and we estimate the strength of the perturbation that causes the "overlap" of the primary islands with rotation number $\frac{1}{n *}$ and $\frac{1}{n^{*+1}}$ in the case $\xi \simeq 1$, where it is known that the "stochasticity" (the dissolution of the invariant curves) begins indeed with the interaction of the $n^{*}$ and $n^{*+1}$ resonances. This "overlap" takes place when (Varvoglis and Papadopoulos 1982a)

$$
\left\{\varepsilon\left|J_{n} *\left\{\left(2 I_{1}\right)^{1 / 2}\right\}\right|\left\{\frac{\xi^{2}}{n *^{2}}+\frac{\varepsilon}{2 I_{1}}\left|J_{n}^{\prime \prime} *\left[\left(2 I_{1}\right)^{1 / 2}\right]\right|\right\}\right\}^{1 / 2} \geqslant \frac{1}{4 n *}
$$

Therefore a trajectory shows stochastic behavior if equ. (6) is satisfied at any of the trajectory points. Equ. (6) reduces to equ. (4) if the term $\frac{\varepsilon}{2 I_{1}} J_{n^{*}}^{\prime \prime}\left\{\left(2 I_{1}\right)^{1 / 2}\right\}$ is ignored compared to $\frac{\xi^{2}}{n^{* 2}}$, and thus the smith and Kaufman result is recovered.

If the chaotic behavior sets on in the degenerate case in the same way as in the non-degenerate one, the stochasticity criterion of equ. (3) for $\xi=0$ would be the limit for $\xi \rightarrow 0$ of the equ. ( 6 )

$$
\frac{\left|J_{n}\left\{\left(2 I_{1}\right)^{1 / 2}\right\} J_{n}^{\prime \prime}\left\{\left(2 I_{1}\right)^{1 / 2}\right\}\right|}{\left(2 I_{1}\right)^{1 / 2}} \geqslant \frac{1}{4 n \varepsilon}
$$

Notice that for $2 I_{1} \gg n^{2}$ equ. (7a) reduces to

$$
\frac{\left|J_{n}\left\{\left(2 I_{1}\right)^{V / 2}\right\}\right|}{\left(2 I_{1}\right)^{1 / 2}} \geqslant \frac{1}{4 \pi \varepsilon}
$$

which is identical to equ. (5). Now it is known (Fukuyama et al 1977 , Karney 1978) that the dissolution of the invariant curves in the degenerate case begins with the interaction of secondary islands, so that the process of taking the limit of equ. (6) cannot be justified. In the special case $v=n+0.5$, however, it has been shown (Hsu 1982) that the cause of the invariant curve dissolution is indeed the interaction of the $n$ and $n+1$ islands, and therefore, at least in this case, the limit of equ. (6) is justified.

It is worth to stress at this point that in the degenerate case the stochasticity criterion turns out to be unique, despite the fact that for various values of the quantity $\delta=|\nu-n|$ the stochasticity sets on following 
different paths. This fact has been recognized in the literature (Karney 1978) and advances the conjecture that perhaps equ. (6) is valid in the whole interval, $0 \leqslant \xi \leqslant 1$, irrespectively of the value of $\delta$ (that is even for $\delta \neq \frac{1}{2}$ ); this conjecture has to be tested numerically to evaluate its usefulness.

In the case of small $\xi$ and large $v$ it has been shown that the above conjecture gives results in agreement with numerical calculations (Varvoglis and Papadopoulos 1982a). In the small $v$ regime we have the interesting situation that equ. (7a) does not fully agree with equ. (5) (old result). Namely, both equs. (5) and (7a) imply that for $v<v_{0}$ the stochastic region in the action space has an upper bound and for $v>v_{0}$ it has an upper and a lower bound, but equ. (5) gives $\nu_{0}=1.5$ while equ. (7a) gives $\nu_{0}=2.5$. This qualitative difference in behavion can be attributed to the fact that for $v<v_{0}$ the origin (of the surface of section) is an unstable periodic orbit, while for $v>v_{0}$ it is a stable one, where $v_{0}$ is equal to 2.5 (Abe et al. 1980, Hsu 1982, Varvoglis and Papadopoulos 1982b); this result is in agreement with equ. (7a).

IV. SUMMARY

The above discussion can be summarized as follows: If a certain degenerate dynamical system can be defined as the limit of a monoparametric family of non-degenerate systems, then it is possible under certain conditions to find its stochasticity criterion as the corresponding limit of the more easily found stochasticity criteria of the non-degenerate systems. In the case of the system of equ. (3) this process not only leads to the (already known) correct answer, but also gives some new information for the behavion of the system in question.

\section{ACKNOWLEDGMENTS}

Part of this work was done while the author was a Research Associate in the Astronomy Program of the University of Maryland. I would like to thank the Solar Terrestrial Theory Group of the Astronomy Program and especially Dr. K. Papadopoulos for the hospitality, the financial support and many useful discussions.

\section{REFERENCES}

Abe, H., Momota, H. and Itatani, R. 1980, Phys. Fluids 23, 2417.

Arnold, V. 1978 Mathematical Methods of Classical Mechanics, New York, Springer, P. 400.

Barbanis, B. 1966, Astron. J. 71, 415.

Berry, M.V. 1978 in Topics in Nonlinear Dynamics, AIP Conference Proceedings 46, New York, AIP.

Chirikov, B.V. 1969, Prepr. 267, Institute of Nuclear Physics, Novosibirsk (Engl. Transl. CERN Transl. 71-40 1971).

Chirikov, B.V. 1979, Phys. Rep. 52, 264.

Fukuyama, A., Momota, H., Itatani, R. and Takizuka, T. 1977, Phys. Rev. Lett. $38,701$.

Greene, J.M. 1980, Ann. N.Y. Acad. Sci. 357, 80. 
Hadjidemetriou, J. and Ichtiaroglou, S. 1983, this volume, p. 141

Hënon, M. and Heiles C. 1964 Astron. J. 69, 73.

Hsu, J.Y. 1982 Phys. Fluids 25, 159.

Kanney, C.F.F. 1978 Phys. Fluids 21, 1584.

Smith, G.R. and Kaufman, A.N. 1978 Phys. Fluids 21, 2230.

Varvoglis, H. and Papadopoulos, K., 1982a, University of Maryland preprint AP82-048.

Varvoglis, H. and Papadopoulos, K., 1982b, Ap.J. Letters (submitted). 